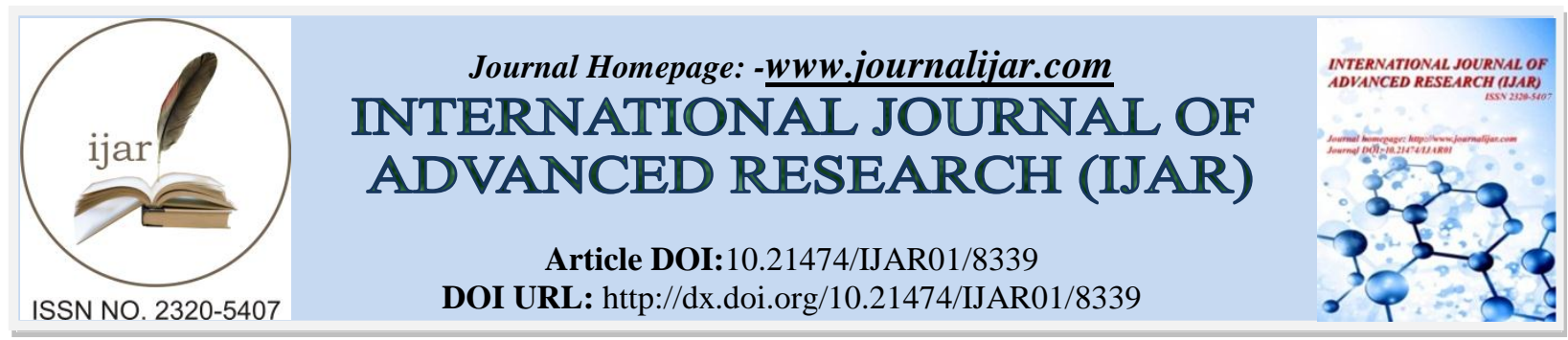

RESEARCH ARTICLE

\title{
LES DESORDRES DE DEVELLOPEMENT SEXUELS (DSD) : UNE PROBLEMATIQUE DE PRISE EN CHARGE.
}

Hanane Moata, Sana Rafi, Ghizlane El Mghari And Nawal El Ansari.

Service d'Endocrinologie, Diabetologie, Maladies metaboliques et Nutrition.

\section{Manuscript Info}

Manuscript History

Received: 08 November 2018

Final Accepted: 10 December 2018

Published: January 2019

Key words:-

anomalies ; développement ; sexuel.

\begin{abstract}
Les différentes anomalies du développement sexuel (DSD) regroupent un ensemble d'atteintes dont le degré de gravité et les conséquences sont variables. La découverte d'une anomalie des organes génitaux sur les échographies anténatales ou lors de l'examen postnatal est une situation rare mais nécessitant une prise en charge urgente et adaptée. Le diagnostic doit être établi le plus tôt possible devant l'urgence vitale en cas de pathologie surrénalienne. Parfois, il est nécessaire de différer la déclaration du sexe de l'enfant le temps des explorations complémentaires.
\end{abstract}

Copy Right, IJAR, 2017,. All rights reserved.

\section{Introduction:-}

Les anomalies de la différenciation sexuelle (DSD) correspondent aux atypies congénitales chromosomiques, gonadiques ou anatomiques du développement sexuel. Elles constituent l'un des plus grands challenges de l'endocrinologue. Leurs causes sont très variées.

La présence de ce type d'anomalie peut constituer une urgence médicale (Hyperplasie congénitale des surrénales ) et sociale. L'impossibilité de donner un sexe à un enfant qui vient de naître est très éprouvant et le choix du sexe doit être le plus rapide possible. Celui-ci est dépendant de multiples facteurs qui sont déterminés par une enquête anatomique, fonctionnelle et psychologique minutieuse dans le but d'établir un diagnostic étiologique précis qui représente le pilier de la prise en charge multidisciplinaire de ces patients. [10 -6]

Classification Des Anomalies De Developpement Sexuel :-

une nouvelle classification a vu le jour Intitulée «anomalies de développement sexuel» ou «Disorder of sexdevelopment»

Corresponding Author:-HananeMoata.

Address:-Service d'Endocrinologie, Diabetologie , Maladies metaboliques et Nutrition. 
Table 1:-Nouvelle nomenclature des anomalies de différenciation sexuelle.

\begin{tabular}{|l|l|}
\hline \multicolumn{1}{|c|}{ Nomenclature ancienne } & \multicolumn{1}{c|}{ Nomenclature proposée } \\
\hline \hline Intersex & DSD \\
\hline \hline Male pseudohermaphrodisme & 46 XY DSD \\
\hline \hline Female Pseudo Hermaphrodite & 46 XX DSD \\
\hline \hline Hermaphrodisme Vrai & Ovotestis DSD \\
\hline \hline$X X$ male ou XX réversion sexuelle & 46 XX testicular DSD \\
\hline \hline$X Y$ réversion sexuelle & 46 XY dysgénésie complète \\
\hline \hline
\end{tabular}

\section{Etiologies :-}

Table 2:-Tableau récapitulatif des principales étiologies des DSD.

\begin{tabular}{|c|c|}
\hline \multirow{2}{*}{$\begin{array}{l}\text { Anomalies } \\
46, \times \times \text { DSD }\end{array}$} & $\begin{array}{l}\text { 1-Hyperolasie congénitale des surrénales: } \\
\text { a-Déficit en } 210 H . \\
\text { b-Déficit en } 11 \text { B OH. } \\
\text { C-Déficit en } 3 \text { B hydroxystéroide déshydrogénase. } \\
\text { d-Déficit en POR }\end{array}$ \\
\hline & $\begin{array}{l}\text { 2-Autres: Hyper-androgénie maternelle, liée à: } \\
\text {-Tumeur ovarienne. } \\
\text {-Hyperplasie virilisante surrénale. } \\
\text {-Prise de médicaments virilisants. }\end{array}$ \\
\hline \multirow{2}{*}{$\begin{array}{l}\text { Anomalies } \\
46, X Y \text { DSD }\end{array}$} & $\begin{array}{l}\text { 1-Déficits des fonctions testiculaires: } \\
\text {-Déficit en AMH. } \\
\text {-Déficit en testostérone. }\end{array}$ \\
\hline & $\begin{array}{l}\text { 2-Perturbation de la sensibilité tissulaire aux androqènes: } \\
\text {-Déficit en } 5 \propto x \text { réductase. } \\
\text {-Déficit du récepteur cytosolique de la testostérone et la DHT. }\end{array}$ \\
\hline \multirow{3}{*}{$\begin{array}{l}\text { Dysgénésies } \\
\text { gonadiques }\end{array}$} & Dysgénésies gonadiques $46 \mathrm{XY}$ : complétes; partielles. \\
\hline & Dysgếnésies gonadiques 46 ×x. \\
\hline & Dysgénésies gonadiques mixtes. \\
\hline
\end{tabular}

\section{Examen clinique:-}

Cet examen clinique initial permet de reconnaître l'ambiguité des OGE selon la classification de Prader qui se compose de cinq stades (fig.1):

1. Stade I:-appareil féminin avec hypertrophie clitoridienne.

2. Stade II:-fusion postérieure des grandes lèvres, orifices urétral et vaginal distincts.

3. Stade III:- la fusion des grandes lèvres se complète et entoure un orifice unique débouchant sur un sinus urogénital.

4. Stade IV:-organe péno-clitoridien recouvert d'un capuchon préputial incomplet, fusion complète des formations labio-scrotales, orifice uro-genital unique situé à la base de la verge en hypospadias scrotal ou pénien postérieur. 
5. Stade V:-verge bien développée avec un orifice uro-génital situé à l'extrémité du gland recouvert par prépuce circonférentiel complet.

Fig1:-Classification de Prader.

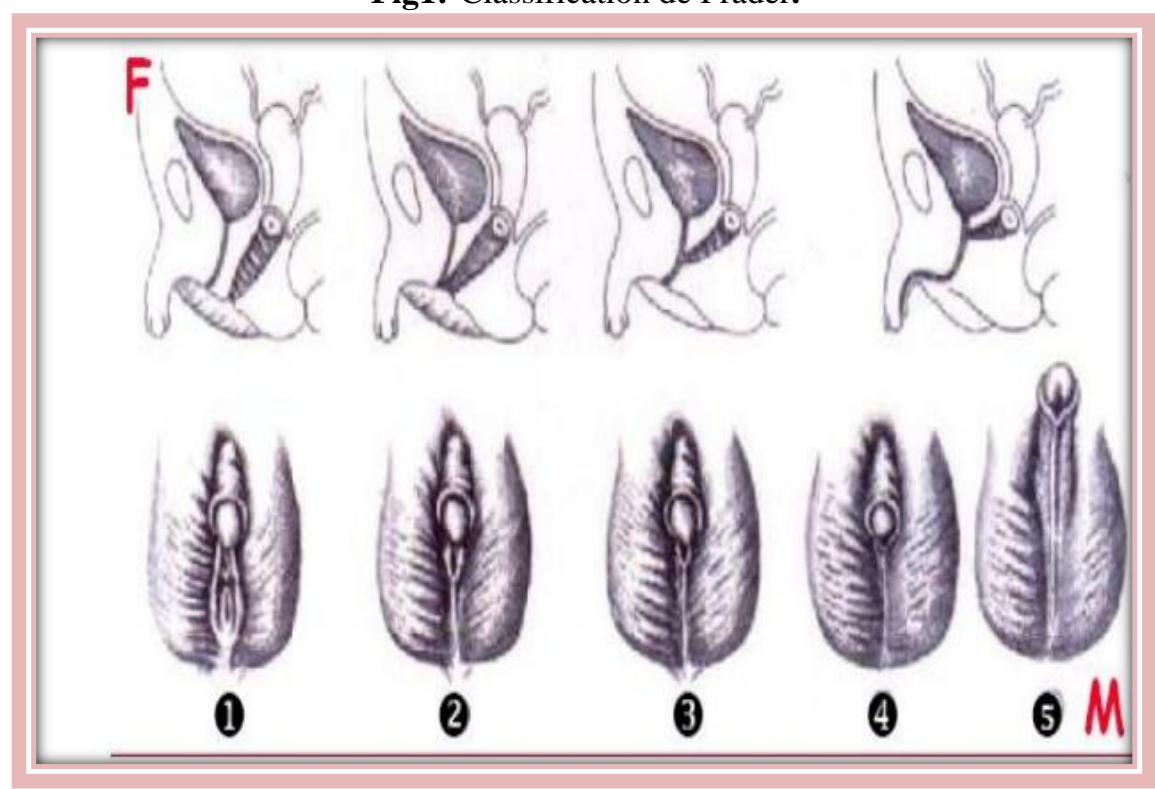

\section{ExamenParaclinique:-}

\section{Biologie:-}

Ionogramme sanguin et urinaire:-

Il objective dans le cas d'un déficit en 21 hydroxylase :

1. une hyponatrémie avec hypernatriurèse (Cliniquement le syndrome de perte de sel apparait du moment où la natrémie est inférieure à $125 \mathrm{meq} / \mathrm{l})$.

2. Une hyperkaliémie

3. l'hypoglycémie et les désordres hydro électrolytiques secondaires à la déshydratation comme l'acidose métabolique. (Dans le déficit en 11 béta $\mathrm{OH}$, en raison de l'excès de synthèse des métabolites minéralocorticoïdes) [9-4 -7]

\section{Explorations hormonales:-}

Nous n'en citerons que l'essentiel:-

1. Dans les anomalies 46,XX DSD, le diagnostic doit être obtenu très rapidement à cause du risque minéralocorticoïde que comportent ces enzymopathies, (blocs les plus fréquents, ceux de la 21 hydroxylase et de la 11 Hydroxylase). Il est plus sûr de doser dans le plasma les concentrations de $17 \mathrm{OH}$ progestérone ou de désoxycortisol (DOC) pour dépister réciproquement le bloc de la 21 hydroxylase et celui de la 11 hydroxylase. La réduction des concentrations plasmatiques de l'un ou l'autre précurseur hormonal sert également de test d'efficacité au freinage de l'hyperplasie cortico-surrénale par la dexaméthasone.

2. Le diagnostic des anomalies 46,XY DSD est plus délicat mais ne requiert pas la même urgence. La conduite des examens biologiques ne peut être codifiée du fait de la grande diversité des troubles, mais le dosage de la testostérone reste la base de ces examens.

3. Le dosage des gonadotrophines plasmatiques, LH et FSH est un temps capital du diagnostic, les gonadotrophines dans les dysgénésies gonadiques sont régulièrement supérieures à la normale avant 6 ans et après 10 ans. L'hypergonadotrophisme permet de séparer, sans ambiguité, les dysgénésies gonadiques de certains hypogonadismes hypothalamo-hypophysaires dont les aspects cliniques sont assez voisins.

4. Dosage de l'hormone anti müllerienne :Son déficit est responsable de la persistance des canaux de muller . [8-5-3] 


\section{Au total : en première intention:-}

Testostérone - 4 androstène-dione - DHEA - $17 \mathrm{OH}$ prégnénoloneDihydro-testostérone - Progestérone - $17 \mathrm{OH}$ progestérone

\section{Radiologie :-}

l'exploration de l'arbre génito-urinaire est assuré principalement par l'échographie ,IRM pelvienne, et la génitographie, l'echographie permet d'affirmer la présence d'un utérus et de visualiser la structure d'ovaires. lagenitographie va préciser le niveau d'implantation de la cavité vaginale sur l'urètre, élément essentiel pour la chirurgie.

Table 3:-bilan paraclinique.

\begin{tabular}{|c|c|c|c|}
\hline \multirow{5}{*}{$\begin{array}{l}\text { Examens } \\
\text { para clinique }\end{array}$} & \multicolumn{3}{|c|}{ lonoqramme sanquin et urininaire } \\
\hline & \multirow{2}{*}{$\begin{array}{l}\text { Bilan } \\
\text { hormonal }\end{array}$} & \multicolumn{2}{|c|}{$\mathrm{XX}: 17 \mathrm{OHP}, \mathrm{T}, \mathrm{DOC}, \mathrm{SDHEA}, \mathrm{FSH}, \mathrm{LH}$} \\
\hline & & \multicolumn{2}{|c|}{$\begin{array}{l}\text { XY: Testostérone, } 170 \mathrm{OH}, \mathrm{DHT}, \Delta 4 \text { androsténedione, } \mathrm{AMH} \text {, } \\
\text { test à l'HCG, FSH, LH }\end{array}$} \\
\hline & $\begin{array}{l}\text { Bilan } \\
\text { radioloqique }\end{array}$ & $\begin{array}{l}\text { Echographie: } \\
\text { Génitographie: }\end{array}$ & $\begin{array}{l}\text { Repérer le siège exact des gonades non } \\
\text { palpables } \\
\text {-Etudier la cavité vaginale. } \\
\text {-Rechercher lempreinte du col utérin. } \\
\text {-Analyser la morphologie et la longueur de } \\
\text { l'urèthre. }\end{array}$ \\
\hline & Coelioscopie & \multicolumn{2}{|c|}{$\begin{array}{l}\text {-Apprécier la nature et le volume des gonades. } \\
\text {-Faire des biopsies, pour avoir le type anatomopathologique } \\
\text { exact. }\end{array}$} \\
\hline $\begin{array}{l}\text { Etude cyto- } \\
\text { Génétique }\end{array}$ & Caryotype : & \multicolumn{2}{|c|}{ XX ou XY ou présence d'une anomalie } \\
\hline
\end{tabular}

\section{Prise En Charge Globale:}

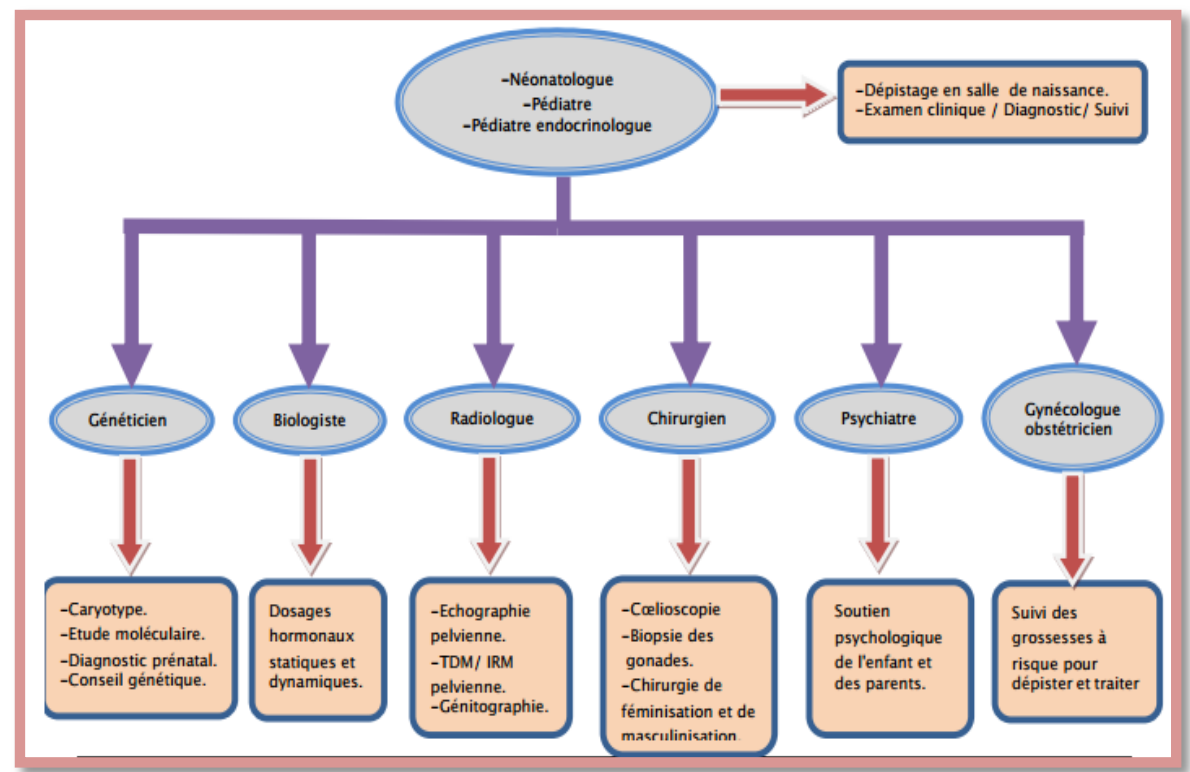




\section{Conclusion:-}

Les désordres de développement sexuel constituent un groupe hétérogène de pathologie dont la compréhension du mécanisme physiopathologique est nécessaire à la prise en charge qui doit être pluridisciplinaire et guidée par des praticiens habitués à ces diagnostics. La constatation d'un DSD à la naissance doit être considérée comme une urgence néonatale.

\section{References:-}

1. C. Pienkowski - a. Cartault ; anomalie du developpement sexuel du nouveau ne-diu maternite novembre 2008

2. F. Roucher - y. Morel · d. Mallet - i. Plotton - v. Tardy .physiopathologie et classification des anomalies du developpementrev. Med. Perinat. (2015) 7:137-146doi 10.1007/s12611-015-0336-6

3. Hughes ia, houk c, ahmed sf, lee pa (2006) consensus statement on management of intersex disorders. Arch dis child91:554-63

4. . Cools m (2014) germ cell cancer risk in dsd patients. Annendocrinol (paris) 75:67-71

5. Cox k, bryce j, jiang j, et al (2014) novel associations in dis-orders of sex development: findings from the i-dsd registry.jclinendocrinolmetab 99:e348-55

6. lee $\mathrm{p}$ etal.consensus of statement on management of intersesx disorders. Pediatrics $118 ; 2:$ e 488-500

7. Huang b et al. Autosomal xx sex reversal caused by duplication of sox9. Am j med genet 1999; 87: 349-353

8. consensus statement on 21 hydroxylase deficiency. J clinendocrinolmetab. 2002; 87:4048-53

9. Mc laughlin dt et al. Sex determination and differenciation. $N$ eng j med. 2004;350:50:367-78

10. Pasterski v, prentice $\mathrm{p}$, hughes ia : consequences of the chicago consensus on disorders of sex development (dsd): current practices in europe. Arch dis child 2010; $95: 618-23$

11. Pathophysiology and classification of disorders of sex development

12. F. Roucher · y. Morel · d. Mallet · i. Plotton · v. Tardy. 